\section{Prácticas parentales y conductas de riesgo del peatón en una muestra de adolescentes chilenos}

\author{
ANDREA C. HERRERA ${ }^{1}$, PAULA B. REPETTO²
}

\section{Parental practices and pedestrian risk behaviors in Chilean adolescents}

\begin{abstract}
Background: Traffic accidents are the second leading cause of death among adolescents and young adults in Chile. However, few studies have examined this behavior among this age group. Parental practices have a great influence on risk behaviors in adolescents, such as substance use, sexuality and violence, among others. Specifically, we propose that these practices will influence pedestrian risk behaviors among adolescents. Aim: To study the role of parental practices such as mother and father support, and behavioral control (monitoring and presence of rules) in pedestrian risk behaviors of teenagers. Material and Methods: A sample of 470 adolescents attending schools in the Metropolitan Region of Santiago, Chile were studied. They answered a self-administered questionnaire in which they were asked about parental practices and pedestrian risk behaviors. Analyses were performed using descriptive and inferential statistics, using multiple regression. Results: Paternal support and the presence of rules were protective factors for pedestrian risky behaviors. However, maternal support or monitoring did not influence these behaviors. Conclusions: Parental practices influence pedestrian behaviors of teenagers. The study provides further evidence for the importance of these practices in the development of behavioral self-regulation.
\end{abstract}

(Rev Med Chile 2014; 142: 975-981)

Key words: Adolescent; Parenting; Risk-taking.

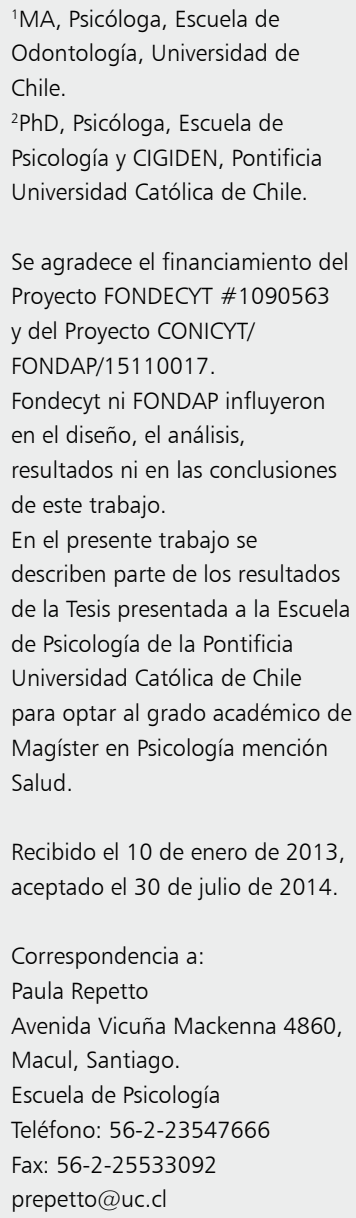

L a imprudencia del peatón es considerada una conducta de riesgo y un importante problema de salud a nivel mundial ${ }^{1}$. Según la Organización Mundial de la Salud, más de un millón de personas mueren anualmente debido a accidentes del tránsito ${ }^{1}$. En el mundo, es la primera causa de mortalidad en el grupo entre los 15 y 19 años y la segunda causa en el grupo de 10 a 14 años $^{2}$. Las estadísticas chilenas son también alarmantes, y los datos de la Comisión Nacional de Seguridad de Tránsito (CONASET) (año 2010) muestran que $41,6 \%$ del total de las víctimas fatales fueron producto de atropellos ${ }^{3}$. De éstas, $56 \%$ fue por imprudencia del peatón ${ }^{3}$. En el grupo entre 15 y 29 años de edad, los accidentes de tránsito son la segunda causa de muerte, con $16,9 \%$ de las muer- tes $^{4}$ y los atropellos constituyen el tercer tipo de siniestro después de las colisiones y los choques ${ }^{5}$. Por esta razón, estudiar los factores que influyen en esta conducta pueden contribuir a mejorar el diseño de estrategias de prevención.

La investigación en psicología en conductas de riesgo del peatón se ha enfocado fundamentalmente en la influencia de los padres en estas conductas en niños preescolares y escolares. Esto se debe que son los padres quienes les enseñan a sus hijos conductas seguras en las calles durante esta etapa del desarrollo ${ }^{6-9}$. Estos estudios muestran que los padres juegan un rol importante en éstas al comunicar las expectativas que tienen respecto a las conductas de seguridad en las calles ${ }^{10}$. También han encontrado que estas conductas cambian y 
que es necesario estudiarlas en etapas posteriores del desarrollo ${ }^{10}$. Sin embargo, no conocemos estudios que hayan examinado las conductas de riesgo del peatón en población adolescente. Esta es la limitación central de la literatura que se aborda con el presente estudio.

El énfasis de la investigación en esta área, con población adolescente, ha estado puesto en estudiar el rol parental en la conducción durante la etapa de novicio ${ }^{11-14}$. Los resultados muestran que las prácticas parentales juegan un rol fundamental en el involucramiento de conductas de riesgo en la conducción de estos jóvenes ${ }^{11,14}$. Los investigadores han encontrado que adolescentes cuyos padres ponen más reglas respecto de la conducción, supervisan la conducta del adolescente y los apoyan, se involucran menos en violaciones de normas del tránsito y en accidentes ${ }^{14}$. Por esa razón, proponemos que las prácticas parentales también influirán en las conductas de los adolescentes como peatones.

Estudiar el rol de las prácticas parentales en las conductas de riesgo del peatón también se fundamenta en los resultados que muestran la importancia que estas prácticas tienen en la prevención de conductas de riesgo en adolescentes ${ }^{15-17}$. Diversos investigadores han mostrado que padres involucrados contribuyen a promover conductas positivas y a prevenir conductas de riesgo en adolescentes ${ }^{18-20}$. En estos estudios, se destaca la importancia que tienen el apoyo parental (aceptación y calidez) ${ }^{21}$ y el control conductual (monitoreo y definición de reglas) en la prevención de conductas de riesgo en adolescentes ${ }^{21}$. Se reconoce que estas prácticas favorecen el desarrollo de la auto-regulación de la conducta y la autonomía, y contribuyen a disminuir la participación de los jóvenes en conductas de riesgo ${ }^{21,22}$.

En resumen, dada la relación encontrada entre prácticas parentales y conductas de riesgo en adolescentes, y particularmente en aquellas relacionadas con la conducción, es que se propone estudiar la influencia de estas prácticas en las conductas de riesgo del peatón en adolescentes. Además, en el presente estudio evaluamos de manera separada el apoyo de la madre y del padre dado que se ha encontrado que el apoyo materno y paterno no tienen la misma influencia en las conductas de riesgo en adolescentes ${ }^{23}$. En el presente estudio, se espera encontrar en los adolescentes participantes, que el mayor apoyo parental y mayor control conductual se asociarán con menos conductas de riesgo del peatón.

\section{Material y Método}

\section{Procedimiento}

Los datos utilizados en este estudio corresponden a la primera evaluación (año 2009) de un estudio longitudinal cuyo objetivo ha sido examinar los patrones de cambio en el consumo de cigarrillos en jóvenes chilenos. Este estudio fue aprobado por los Comités de Ética de la Escuela de Psicología de la Pontificia Universidad Católica de Chile y de Bioética de FONDECYT, cumpliendo así con la Declaración de Helsinki. Todos los participantes firmaron un asentimiento (menores de edad) o consentimiento informado (mayores de 18 años) para poder participar en el estudio. Los padres de los menores de edad autorizaron la participación de sus hijos en el estudio.

Una vez obtenida la autorización de las instituciones educacionales, de los padres y de los participantes, se llevó a cabo la aplicación del cuestionario. El cuestionario fue aplicado de manera colectiva, en sala de clases, por evaluadores entrenados. En este estudio sólo se incluyó a menores de edad.

\section{Instrumentos}

Todas las escalas utilizadas en el estudio han sido usadas con anterioridad en población chilena o similar a la evaluada en éste.

Para medir apoyo parental se utilizó la Escala de Apoyo Parental ${ }^{24}$. Esta tiene10 preguntas con un formato de respuesta tipo Likert de 5 puntos $(0$ nunca a 4 casi siempre) y evalúa el apoyo percibido de la madre y el padre por separado. Un ejemplo de ítem es "Te resulta fácil expresar tus sentimientos a tu padre (madre)".

Para evaluar Control Conductual se usaron dos cuestionarios. Primero, el Cuestionario de Reglas Parentales ${ }^{16}$, que pregunta si los padres tienen reglas claras para 11 temas. Un ejemplo de pregunta, “¿Tienen tus padres reglas claras con cómo usas tu tiempo libre?". Las alternativas de respuestas se codifican con 0 para no y 1 para sí. Segundo, la Escala de Monitoreo Parental ${ }^{25}$. Se utilizaron cuatro ítems que miden monitoreo y cuyas alternativas de respuestas van desde nunca (0) a casi siempre (4). Un ejemplo de pregunta 
Prácticas parentales y conductas de riesgo del peatón - A. Herrera et al

es ¿Cuán a menudo tu papá o mamá te preguntan a dónde vas?".

Las conductas de riesgo del peatón fueron medidas usando la Escala de Comportamiento Peatonal ${ }^{26}$ que incluye 13 preguntas referidas a la frecuencia de comportamientos transgresores, errores o lapsus que un peatón puede cometer al caminar o movilizarse por la ciudad, por ejemplo, ¿Cruzo semáforos aún cuando no están con luz ver$d e$ ?. Las alternativas de respuesta van desde nunca (1) a siempre (6). De la escala fueron eliminados dos ítems cuyas cargas factoriales fueron bajas (menores a 0,4 ). El análisis factorial fue realizado usando componentes principales y rotación Oblimin con Kaiser y mostró que la mejor solución incluye dos factores. Uno, de las conductas del peatón relacionadas con el cruce de calles (Peatón Calle), por ejemplo, ¿Cruzo semáforos aún cuando no están con luz verde? y un segundo factor (Peatón Vereda) que incluye conductas relacionadas con el desplazamiento por la vereda, por ejemplo, Olvido transitar por la derecha en las veredas.

Finalmente, para recolectar la información demográfica de los participantes, se les preguntó por su fecha de nacimiento, sexo y curso.

\section{Participantes}

Los participantes del estudio fueron 470 estudiantes de séptimo básico a cuarto año medio de tres establecimientos educacionales (dos particulares subvencionados y uno municipal) del área Suroriente de la Región Metropolitana (49,1\% hombres, edad promedio 14,4 años, DE 2,6; rango 9 a 17 años) que aceptaron participar en el estudio.
La selección de la muestra fue por conveniencia y se trabajó con adolescentes de las escuelas que accedieron a participar.

Para los análisis eliminamos 29 participantes que tenían datos incompletos en algunas de las variables incluidas en el estudio. Al comparar a los adolescentes incluidos de los excluidos encontramos que los excluidos eran más jóvenes $\left(\mathrm{F}_{1,476}\right.$ $=6,67, \mathrm{p}<0,01)$ y reportaban mayor apoyo de parte de su padre $\left(F_{1,476}=6,00, p<0,05\right)$. No encontramos diferencias entre incluidos y excluidos en ninguna de las otras variables.

\section{Análisis estadísticos}

Todos los análisis estadísticos se realizaron aceptando una probabilidad de error tipo I de 0,05. Se realizaron análisis descriptivos de los puntajes obtenidos en las distintas escalas incluidas en el estudio y análisis inferenciales para examinar las hipótesis propuestas usando regresión múltiple. Se utilizó software SPSS versión 17 para todos los análisis.

\section{Resultados}

\section{Resultados descriptivos}

En la Tabla 1 se resumen los resultados de los análisis descriptivos de las escalas utilizadas (promedio, desviación estándar y consistencia interna). Se realizaron correlaciones parciales (Spearman) entre todas las variables que se presentan en la Tabla 2. Estos resultados muestran una asociación negativa entre las conductas de

\section{Tabla 1. Estadísticos descriptivos de las escalas de prácticas parentales y conductas de riesgo} del peatón de toda la muestra $(n=470$, medición año 2009)

\begin{tabular}{|lcccc|}
\hline Variables & Promedio & $\begin{array}{c}\text { Desviación } \\
\text { estándar }\end{array}$ & $\begin{array}{c}\text { Rango de } \\
\text { puntajes }\end{array}$ & $\begin{array}{c}\text { Consistencia interna } \\
\text { (Alfa de Cronbach) }\end{array}$ \\
Apoyo madre & 2,89 & 0,91 & $0-4$ & 0,85 \\
Apoyo padre & 2,19 & 1,00 & $0-4$ & 0,84 \\
Control conductual & 1,79 & 0,87 & $0-4$ & 0,62 \\
$\quad$ Monitoreo & 0,72 & 0,24 & $0-1,09$ & 0,75 \\
Reglas & 2,58 & 1,04 & $1-6$ & 0,57 \\
Peatón en la calle & 3,75 & 1,34 & $1-6$ & 0,59 \\
\hline Peatón en la vereda & & & \\
\hline
\end{tabular}


Tabla 2. Matriz de correlaciones entre cada una de las escalas de prácticas parentales y conductas de riesgo del peatón $(n=470$, medición año 2009)

\begin{tabular}{|lcccccc|}
\hline & Apoyo madre & Apoyo padre & Monitoreo & Reglas & Peatón calle & Peatón vereda \\
Apoyo madre & 1,000 & & & & & \\
Apoyo padre &, $302^{* *}$ & 1,000 & & & & \\
Monitoreo &, 079 &,- 023 & 1,000 & & & \\
Reglas &, $096^{*}$ &, $196^{* *}$ &, $281^{* *}$ & 1,000 & & \\
Peatón calle &,$- 108^{*}$ &,$- 143^{* *}$ &,- 041 &,$- 222^{* *}$ & 1,000 &, $488^{* *}$ \\
Peatón vereda &,$- 095^{*}$ &,$- 109^{*}$ &,- 019 &,$- 162^{*}$ & 1,000 \\
\hline
\end{tabular}

** La correlación es significativa al nivel 0,01 (bilateral). * La correlación es significativa al nivel 0,05 (bilateral).

Tabla 3. Resultados de los modelos de regresión para las variables peatón vereda y peatón calle y la influencia de las prácticas parentales ( $n=470$, medición año 2009)

\begin{tabular}{|c|c|c|c|c|c|}
\hline & \multicolumn{2}{|c|}{ Coeficientes no estandarizados } & \multirow{2}{*}{$\begin{array}{c}\text { Coeficientes } \\
\text { estandarizados } \\
\text { Beta }\end{array}$} & \multirow[b]{2}{*}{$\mathbf{t}$} & \multirow[b]{2}{*}{ Sig. } \\
\hline & B & Error típico & & & \\
\hline \multicolumn{6}{|l|}{ Peatón vereda } \\
\hline (Constante) & 4,558 & 313 & & 14,569 &, 000 \\
\hline Apoyo del padre &,- 150 &, 062 &,- 117 & $-2,420$ &, 016 \\
\hline Apoyo de la madre &,- 100 & ,073 &,- 070 & $-1,368$ & 172 \\
\hline Monitoreo & 173 & 101 & ,088 & 1,701 & 090 \\
\hline Reglas & $-1,091$ & ,255 &,- 206 & $-4,274$ &, 000 \\
\hline \multicolumn{6}{|l|}{ Peatón calle } \\
\hline (Constante) & 4,448 &, 327 & & 13,615 &, 000 \\
\hline Apoyo del padre &,- 139 &, 065 &,- 104 & $-2,147$ &, 032 \\
\hline Apoyo de la madre &,- 103 &, 076 &,- 070 & $-1,354$ & 176 \\
\hline Monitoreo & , 174 & 106 & ,086 & 1,645 & 101 \\
\hline Reglas &,- 897 & 267 &,- 164 & $-3,366$ & ,001 \\
\hline
\end{tabular}

riesgo del peatón con el apoyo de la madre y el apoyo del padre, y con la presencia de reglas. No se encontró una asociación entre monitoreo y estas conductas de riesgo.

\section{Prácticas parentales $y$ conductas de riesgo}

\section{del peatón}

Para evaluar la relación entre apoyo parental, control conductual (monitoreo y presencia de reglas) y conductas de riesgo del peatón se realizaron dos análisis de regresión múltiple, usando pasos sucesivos (stepwise) para cada variable dependiente. Los resultados muestran que mayor apoyo del padre y más reglas se asocian con menores conductas de riesgo del peatón tanto en el caso de la subescala de peatón calle $\left(\mathrm{F}_{4,444}=7,661, \mathrm{p}<0,001\right.$, $\mathrm{R} 2=0,056)$, como de la subescala de peatón vereda $\left(\mathrm{F}_{4,445}=5,319, \mathrm{p}<0,001, \mathrm{R} 2=0,037\right)$ (Tabla 3). El apoyo de la madre y el monitoreo no se asocian con estas conductas.

\section{Discusión}

El objetivo del presente estudio fue evaluar distintas prácticas parentales y su relación con las conductas de riesgo del peatón en adolescentes. Los resultados obtenidos confirman las hipótesis propuestas y muestran que las prácticas parentales influyen en las conductas de riesgo del peatón. 
Nuestros resultados sugieren que adolescentes que reportan tener mayor apoyo por parte del padre y cuyos padres imponen más reglas, presentan menos conductas de riesgo como peatones.

Estos resultados son consistentes con la literatura que muestra el rol que juegan los padres en el control de las conducta y de las actitudes del adolescente, así como en el desarrollo de la autonomía y la auto-regulación conductual. Padres que imponen reglas claras tienen hijos que participan menos en conductas de riesgo ${ }^{21,27}$. También muestran que el apoyo del padre jugaría un rol importante en este proceso. A través del apoyo, los padres transmiten reglas específicas relacionadas con conductas particulares, las cuales podrían estar relacionadas con la seguridad del tránsito. El apoyo parental juega un rol importante en la medida que permite un involucramiento con el adolescente que favorece la transmisión de normas o conductas esperadas al momento de cruzar la calle y al promover la competencia y el autocontrol $^{21,27}$. Esto es consistente con los estudios que muestran la importancia del apoyo de los padres para el desarrollo de la autonomía y la regulación de la conducta ${ }^{21}$.

Los resultados que muestran la importancia de la presencia de reglas en las conductas de riesgo menores del peatón, son coherentes con la perspectiva de la socialización, que enfatiza la transmisión de los valores parentales al adolescente a través de estrategias de control conductual que favorecen el desarrollo de la autonomía y auto-regulación conductual ${ }^{21}$. También son consistentes con lo planteado por Morrongiello y cols., quienes destacan el rol que juega el establecimiento de reglas y pautas de conducta en las conductas de riesgo del peatón en los hijos en etapas más tempranas del desarrollo ${ }^{10}$.

Los resultados, respecto al rol de reglas en estas conductas, también pueden ser entendidos a partir de los hallazgos de Darling y cols., quienes han estudiado prácticas parentales en adolescentes chilenos ${ }^{16}$. Estos autores reportan que existiría un acuerdo entre adolescentes y padres chilenos que permite que los padres puedan controlar a los jóvenes en temas de seguridad, pero no así en temas de dominio personal. Es decir, los adolescentes legitiman y respetan que los padres impongan reglas en temas de seguridad, lo que explicaría la relación entre reglas y las conductas de seguridad del peatón en adolescentes chilenos. Este resultado es relevante porque muestra un ámbito que puede contribuir al desarrollo de estrategias de prevención de estas conductas de riesgo en adolescentes.

Algunos resultados obtenidos no son consistentes con las hipótesis propuestas. No encontramos una asociación entre el apoyo de la madre y el monitoreo con las conductas de riesgo del peatón. Es puede deberse a que, en general, el apoyo materno reportado en esta muestra fue alto y tuvo una varianza menor que el apoyo del padre, haciendo más difícil encontrar efectos. Sin embargo, estos resultados son consistentes con estudios en los cuales no se ha encontrado una relación entre apoyo de la madre y conductas de riesgo en adolescentes, pero sí una influencia del apoyo del padre ${ }^{28}$, y que muestran el rol diferencial que padres y madres tienen en la socialización de sus hijos ${ }^{29}$. Nuestros resultados muestran que estudiar de manera separada el apoyo de la madre y del padre puede contribuir a comprender mejor el rol que cada uno asume en el desarrollo de las conductas de riesgo.

Los resultados en relación al monitoreo parental tampoco son consistentes con lo esperado. Esto podría deberse a la medición utilizada, la cual es una medida global de monitoreo. En estudios sobre conducción de novicios no se han encontrado efectos cuando el monitoreo se ha medido en términos globales y sí lo son cuando el monitoreo es medido en relación a esta conducta y durante su ejecución ${ }^{11,12}$. En estudios futuros se debiera incluir preguntas específicas de monitoreo de los padres con relación a las conductas peatonales.

Futuros estudios debieran explorar el rol de los pares en esta conducta. Se reconoce la creciente influencia que adquieren los pares durante la adolescencia y en las conductas de riesgo ${ }^{30,31}$. Los adolescentes pasan cada vez más tiempo con sus pares y es probable que muchas de estas conductas ocurran con ellos presentes. Por lo tanto, explorar su influencia podría ayudar a comprender mejor esta conducta.

Finalmente, es importante mencionar las limitaciones del presente estudio. Entre estas se encuentran el tipo de muestra, la cual es intencionada y no necesariamente representa a la población chilena. Sin embargo, según los datos del MINEDUC, estas escuelas se asemejan a otras escuelas con similares características sociodemográficas del país, considerando su rendimiento en la prueba $\mathrm{SIMCE}^{32}$. Por otro lado, el uso de auto-reporte 
en la medición es una limitación porque puede no reflejar de manera precisa esta conducta. De todas maneras, se usaron escalas validadas y usadas ampliamente por los investigadores en este campo en adolescentes chilenos y se aseguró la confidencialidad a los participantes. Además, los resultados son consistentes con lo registrado en la literatura lo que sugiere que, a pesar de estas limitaciones, son resultados que pueden ser considerados como válidos y que permiten contribuir a comprender mejor estas conductas.

Nuestros resultados muestran la importancia que tienen las prácticas parentales en las conductas de riesgo del peatón en adolescentes, y muestran que favorecer el involucramiento del padre y definir reglas orientadas a regular la conducta del adolescente, puede ayudar a prevenir estas conductas de riesgo.

\section{Referencias}

1. World Health Organization. Global Status Report on Road Safety: Time for Action. Geneva: WHO; 2009.

2. World Health Organization. Youth and Road Safety. Geneva: WHO; 2007.

3. CONASET. Atropellos ocurridos en Chile: Diagnóstico año 2010. In: CONASET, ed. Santiago, Chile; 2010.

4. CONASET. Los Jóvenes en el Tránsito (período 20002010). In: CONASET, ed. Santiago, Chile; 2010.

5. CONASET. Atropellos en Chile año 2009. Santiago, Chile: CONASET; 2009.

6. Morrongiello BA, Barton BK. Child pedestrian safety: parental supervision, modeling behaviors, and beliefs about child pedestrian competence. Accid Anal Prev 2009; 41 (5): 1040-6.

7. Barton BK, Schwebel DC, Morrongiello BA. Brief report: Increasing children's safe pedestrian behaviors through simple skills training. J Pediatr Psychol 2007; 32 (4): 475-80.

8. Holland CA, Hill R, Cooke R. Understanding the role of self-identity in habitual risky behaviours: pedestrian road-crossing decisions across the lifespan. Health Educ Res 2009; 24 (4): 674-85.

9. Evans D, Norman P. Predicting adolescent pedestrians' road-crossing intentions: an application and extension of the Theory of Planned Behaviour. Health Educ Res 2003; 18 (3): 267-77.

10. Morrongiello BA, Corbett M, Bellissimo A. "Do as I say, not as I do": family influences on children's safety and risk behaviors. Health Psychol 2008; 27 (4): 498-503.
11. Simons-Morton BG, Ouimet MC, Catalano RF. Parenting and the young driver problem. Am J Prev Med 2008; 35 (3 Suppl): S294-303.

12. Prato CG, Toledo T, Lotan T, Taubman-Ben-Ari O. Modeling the behavior of novice young drivers during the first year after licensure. Accid Anal Prev 2010; 42 (2): 480-6.

13. Elliott MR, Jacobsohn L, Winston FK, Ginsburg KR. Determining subgroups of teens for targeted driving injury prevention strategies: a latent class analysis approach. Traffic Inj Prev 2012; 13 (3): 258-64.

14. Ginsburg KR, Durbin DR, García-Espana JF, Kalicka EA, Winston FK. Associations between parenting styles and teen driving, safety-related behaviors and attitudes. Pediatrics 2009; 124 (4): 1040-51.

15. Steinberg L, Morris AS. Adolescent development. Annu Rev Psychol 2001; 52: 83-110.

16. Darling N, Cumsille P, Peña-Alampay L. Rules, legitimacy of parental authority, and obligation to obey in Chile, the Philippines, and the United States. New Dir Child Adolesc Dev 2005 (108): 47-60.

17. Florenzano UR, Valdés CM, Cáceres CE, Santander RS, Aspillaga HC, Musalem AC. [Relation between suicidal ideation and parenting styles among a group of Chilean adolescents]. Rev Med Chile 2011; 139 (12): 1529-33.

18. Haggerty KP, Fleming CB, Catalano RF, Harachi TW, Abbott RD. Raising healthy children: examining the impact of promoting healthy driving behavior within a social development intervention. Prev Sci 2006; 7 (3): 257-67.

19. Haggerty KP, Skinner ML, MacKenzie EP, Catalano RF. A randomized trial of Parents Who Care: effects on key outcomes at 24-month follow-up. Prev Sci 2007; 8 (4): 249-60.

20. Fletcher AC, Steinberg L, Williams-Wheeler M. Parental influences on adolescent problem behavior: revisiting Stattin and Kerr. Child Dev 2004; 75 (3): 781-96.

21. Barber BK, Stolz HE, Olsen JA. Parental support, psychological control, and behavioral control: assessing relevance across time, culture, and method. Monogr Soc Res Child Dev 2005; 70 (4): 1-137.

22. Barber BK, Maughan SL, Olsen JA. Patterns of parenting across adolescence. New Dir Child Adolesc Dev 2005 (108): 5-16.

23. Furman W, Buhrmester D. Age and sex differences in perceptions of networks of personal relationships. Child Dev 1992; 63 (1): 103-15.

24. Procidano ME, Heller K. Measures of perceived social support from friends and from family: three validation studies. Am J Community Psychol 1983; 11 (1): 1-24. 
Prácticas parentales y conductas de riesgo del peatón - A. Herrera et al

25. Darling N, Cumsille P, Martínez ML. Adolescents' as active agents in the socialization process: legitimacy of parental authority and obligation to obey as predictors of obedience. J Adolesc 2007; 30 (2): 297-311.

26. Moyano E. Teoría del Comportamiento Planificado e Intención de Infringir Normas de Tránsito en Peatones. Estudios de Psicología 1997; 2: 335-48.

27. Wills TA, Resko JA, Ainette MG, Mendoza D. Role of parent support and peer support in adolescent substance use: a test of mediated effects. Psychol Addict Behav 2004; 18 (2): 122-34.

28. Piko B. Perceived social support from parents and peers: which is the stronger predictor of adolescent substance use? Subst Use Misuse 2000; 35 (4): 617-30.
29. McElwain NL, Halberstadt AG, Volling BL. Mother-and father-reported reactions to children's negative emotions: relations to young children's emotional understanding and friendship quality. Child Dev 2007; 78 (5): 1407-25.

30. Viner RM, Ozer EM, Denny S, Marmot M, Resnick M, Fatusi A, et al. Adolescence and the social determinants of health. Lancet 2012; 379 (9826): 1641-52.

31. Brechwald WA, Prinstein MJ. Beyond Homophily: A Decade of Advances in Understanding Peer Influence Processes. J Res Adolesc 2011; 21 (1): 166-79.

32. Ministerio de Educación C 2014; Pages. Accessed at MINEDUC at http://www.mime.mineduc.cl/mvc/mime/ 10 de enero de 2014. 